\section{Preliminary Findings of Long-Term Neurodevelopmental Outcomes of Infants Treated With Intravenous Fat Emulsion Reduction for the Management of Parenteral Nutrition-Associated Cholestasis}

Journal of Parenteral and Enteral Nutrition

Volume 39 Number

January $201534-46$

(C) 2014 American Society

for Parenteral and Enteral Nutrition DOI: $10.1177 / 0148607114551965$

jpen.sagepub.com

hosted at

online.sagepub.com

\author{
Allison B. Blackmer, PharmD, BCPS ${ }^{1,2}$; Seth Warschausky, $\mathrm{PhD}^{3}$; Sabina Siddiqui, $\mathrm{MD}^{4}$; \\ Kathleen B. Welch, MS, MPH ${ }^{5}$; Karolyn Horn, PharmD ${ }^{6}$; Ashley Wester, PharmD ${ }^{6}$; \\ Micah Warschausky ${ }^{3}$; and Daniel H. Teitelbaum, MD $^{4}$
}

\begin{abstract}
Introduction: Parenteral nutrition-associated cholestasis (PNAC) is linked with the administration of soybean-based intravenous fat emulsion (IVFE). IVFE reduction (IFER) may be an effective management strategy for PNAC; however, long-term associated neurodevelopmental outcomes (NDOs) for infants undergoing IFER have not been measured previously. This single-institution, prospective study examined the risk for negative NDOs and key predictors of NDOs associated with IFER. Methods: Patients (2-5 years) treated with soybean-based IFER as neonates underwent NDO measurements, including Ages and Stages Questionnaires-3 (ASQ-3), Parents' Evaluations of Developmental Status (PEDS), and Behavior Assessment System for Children, Second Edition Preschool, Parent (BASC-2 PRS-P). The relationship between NDOs and predictive variables was evaluated. Results: A total of 25 children had a complete PEDS survey, and 17 were found to be "not at risk." The BASC-2 PRS-P evaluation ( $\mathrm{n}=18$ patients) showed that all 4 composite domains fell within the normative developmental range, and $67 \%-89 \%$ of patients were observed to be "typically developing." For the primary outcome measure, ASQ-3, 82.4\%-94.4\% of patients were "not at risk." Logistical regression analyses were performed to examine risk factors contributing to negative NDOs. Of children completing all NDO studies, IFER-related variables (eg, development of essential fatty acid deficiency, duration of IFER, and mean IVFE dose) were not found to be predictors of adverse NDOs. Conclusions: This study represents the first report of NDOs in pediatric patients treated with IFER. IFER-treated patients score within the normative range most of the time. IFER-related variables were not found to be associated with negative NDOs. The results set the stage for a larger, multicenter, prospective study. (JPEN J Parenter Enteral Nutr. 2015;39:34-46)
\end{abstract}

\title{
Keywords
}

neurodevelopment; lipid minimization; cholestasis; infants

\section{Clinical Relevancy Statement}

Currently, the only intravenous fat emulsion that is approved for use in pediatric patients in the United States by the Food and Drug Administration is soybean oil based. However, the long-term administration of soybean-based intravenous fat emulsion to parenteral nutrition (PN)-dependent patients has been shown to be an independent risk factor for the development of PN-associated liver disease. One strategy that has been suggested to reduce this risk is the reduction in dose of soybean-based fat emulsion. However, the long-term neurodevelopmental outcomes associated with this strategy are unknown to date. This single-institution study represents the first published report of neurodevelopmental outcomes in pediatric patients treated with reduced doses of soybean-based fat emulsion. The neurodevelopmental outcomes were within the normative range most of the time, and variables associated with dose reductions were not predictive of poor neurodevelopmental outcomes. This preliminary study calls for further research to conclusively determine the influence of soybean-based fat emulsion dose reduction on long-term neurodevelopmental outcomes.
From the ${ }^{1}$ Department of Clinical, Social and Administrative Sciences, University of Michigan College of Pharmacy, Ann Arbor; ${ }^{2}$ Department of Pharmacy Services, University of Michigan Health System, Ann Arbor; ${ }^{3}$ Department of Physical Medicine and Rehabilitation, University of Michigan Health System, Ann Arbor; ${ }^{4}$ Department of Surgery, C. S. Mott Children's Hospital, University of Michigan, Ann Arbor; ${ }^{5}$ School of Public Health, University of Michigan, Ann Arbor; and ${ }^{6}$ College of Pharmacy, University of Michigan, Ann Arbor.

Financial disclosure: This research was funded by the C. Richard Fleming Grant from the American Society for Parenteral and Enteral Nutrition Rhoads Research Foundation.

Received for publication December 17, 2013; accepted for publication August 27, 2014.

This article originally appeared online on October 7, 2014.

Corresponding Author:

Allison B. Blackmer, PharmD, BCPS, University of Colorado Anschutz Medical Campus, Skaggs School of Pharmacy and Pharmaceutical Sciences, Mail Stop C238. 12850 E. Montview Blvd. Aurora, CO 80045, USA.

Email: allison.blackmer@ucdenver.edu 


\section{Background/Introduction}

Infants, particularly those with surgical conditions, are often dependent on long-term parental nutrition (PN) during a critical period of growth and development. While PN offers a lifesustaining therapy, there are multiple known infectious and metabolic complications from prolonged $\mathrm{PN}$ use, including the development of PN-associated cholestasis (PNAC), which is associated with significant morbidity and mortality. ${ }^{1-3}$ Specifically, in a study of surgical neonates, nearly one-third of patients dependent on long-term PN died due to the development of PNAC, ${ }^{4}$ and in a study of children with short bowel syndrome, PNAC was the greatest risk factor for mortality. ${ }^{5}$ The incidence of PNAC ranges from $7.4 \%-84 \%$; this variable reported incidence is attributed to study heterogeneity. ${ }^{1,6}$ While more recent survival rates for PNAC have improved, the disease process remains significant. Several risk factors for the development of PNAC have been identified, including prolonged PN administration, lack of enteral feedings, prematurity, episodes of sepsis, and specific components of PN itself. $^{2,7,8}$ The administration of soybean-based intravenous fat emulsion (IVFE) has been identified as an independent risk factor for the development of PNAC. ${ }^{9,10}$ Soybean-based IVFE contain high levels of $\omega-6$ fatty acids as well as phytosterols, which have been linked to the development of PNAC. ${ }^{11}$ Given this information, several strategies have been used to limit the delivery of soybean-based IVFE, including the use of intravenous (IV) fish oil or alternative, combination-based fat emulsions, and IVFE reduction (IFER). ${ }^{12,13}$

The approach of IFER involves limiting the dose of soybean-based IVFE to $\leq 1 \mathrm{~g} / \mathrm{kg} / \mathrm{d}$. The benefit of IFER was first described in the adult literature in 1982, but it was not until 2000 that a similar approach was reported in a pediatric population. ${ }^{14,15}$ Since then, several studies have evaluated the use of IFER as a management approach for the prevention and treatment of PNAC. ${ }^{16-20}$ At our institution, infants receiving longterm PN (ie, PN duration $>2$ weeks) who develop PNAC are treated with IFER. This approach has been used at our center since 2005 , with patients receiving doses ranging from $1 \mathrm{~g} / \mathrm{kg}$ twice per week to $1 \mathrm{~g} / \mathrm{kg} / \mathrm{d}$, depending on clinical factors such as growth, development, nutrition status, and the development of essential fatty acid deficiency.

While IFER appears to offer potential benefit in the setting of PNAC, questions remain about the safety of this management approach, specifically with respect to the associated neurodevelopmental outcomes (NDOs), since limiting IVFE may also limit the essential fatty acids necessary for appropriate neuro- and behavioral development. Appropriate caloric intake and provision of essential fatty acids are of specific significance during preterm and term neonatal and infant development, when processes such as brain development and organogenesis take place. ${ }^{21}$ Several randomized controlled studies have attempted to show the benefit of administering certain fatty acids to promote age-appropriate neurodevelopment and treat neurocognitive disorders with mixed outcomes. ${ }^{22-27}$ However, to date, there are no data available describing the long-term neuro- and behavioral developmental outcomes in infants treated with IFER.

A number of validated parent rating instruments have been developed to screen young children for developmental risks, with longstanding evidence that parent-identified concerns are predictive of developmental delays across a range of developmental domains. ${ }^{28}$ Two of the most commonly used instruments are the Ages and Stages Questionnaires (ASQ) and the Parent Evaluation of Developmental Status (PEDS) ${ }^{29-31}$ These types of parent-completed instruments have been shown to have moderate to high sensitivity (PEDS, 74\%; ASQ-3, 82\%) to developmental delays identified with standardized measures of development, cognition, and adaptive behavior but have variable specificity (PEDS, 64\%; Ages and Stages Questionnaires-3 [ASQ-3], 78\%) across age groups, particularly in low-risk samples. ${ }^{32}$ For example, in a low-risk sample, there is evidence of discordant identification in approximately one-third of cases. ${ }^{33}$ Specificity is higher in high-risk samples, however. ${ }^{34}$ Thus, in samples with unknown risk for developmental delay, there may be value in using a set of instruments, rather than a single developmental screening instrument. Complementary to measures of risk for developmental delay, there has been longstanding interest in parent-completed instruments that aid in early identification of risk for psychological and social developmental disorders. ${ }^{28}$ One of the most commonly used parent-completed measures of behavior is the Behavior Assessment System for Children, Second Edition (BASC-2), which includes measurement of adaptive behaviors that can add an important dimension to risk identification. ${ }^{35,36}$ Thus, to address the need for a robust assessment of NDOs, these 3 tools (ie, ASQ-3, PEDS, and BASC-2) were selected for the NDO evaluation.

Based on the excellent long-term outcomes clinically observed of many of our patients treated with IFER, we hypothesized that NDOs would not be adversely affected with the use of IFER. This single-institution, prospective study examined the risk for cognitive and adaptive delays and behavioral concerns, as well as key predictors of NDOs, in pediatric patients previously treated with IFER. To our knowledge, this is the first report of this type.

\section{Methods}

This study was conducted in accordance with the ethical standards set forth and approved by our institutional review board, and all parents or guardians gave consent prior to conducting these studies. This was a single-institution study with the primary aim of examining the NDOs of pediatric patients between 2 and 5 years of age who were previously treated with IFER during infancy. Specifically, the study further examined the risk for cognitive and adaptive delays and behavioral disorders and key predictors of neurodevelopmental and behavioral outcomes in this potentially at-risk population. 
The method for the use of IFER at this institution has been described previously. ${ }^{16,17}$ In brief, IFER consisted of the reduction of IVFE from the standard $3 \mathrm{~g} / \mathrm{kg} / \mathrm{d}$ to $\leq 1 \mathrm{~g} / \mathrm{kg} / \mathrm{d}$; initial doses ranged from $1 \mathrm{~g} / \mathrm{kg}$ twice per week to $1 \mathrm{~g} / \mathrm{kg} / \mathrm{d}$ and averaged $1 \mathrm{~g} / \mathrm{kg} 3$ times per week. IVFE doses were increased if poor growth was observed or if biochemical and physical signs and symptoms of essential fatty acid deficiency were present. IFER was initiated after at least 2 weeks of continuous PN in infants who developed PNAC, as defined by a direct bilirubin $\geq 2 \mathrm{mg} / \mathrm{dL}$ for 2 consecutive weeks.

To be eligible for inclusion, the study participant must have been living at the time of the study and had received IFER treatment as an infant between August 1, 2005, and August 31, 2011. Inclusion criteria comprised patients who were previously treated with IFER during the neonatal period and over at least the first several months of life. Patients also had to be between 2 and 5 years of age at the time of NDO measurement. Patients were excluded if they met 1 or more of the following criteria: (1) a congenital or chromosomal anomaly, (2) a major congenital metabolic disorder, (3) hypoxic ischemic encephalopathy or other major congenital defects of the brain, or (4) a major seizure disorder or cerebral palsy. No exclusions were made on the basis of sex or race. The neurodevelopmental survey tools were conducted in the English language, and therefore the ability to speak and read English was necessary. The study range of 2 to 5 years of age was based on the fact that the neurodevelopmental survey tools used in this study are designed to measure outcomes in children within specific age groups and because outcomes of interest may not manifest until infants reach the preschool or school age. NDOs were evaluated by 3 validated outcomes tools: (1) ASQ-3, (2) PEDS, and (3) BASC-2 Preschool, Parent (BASC-2 PRS-P).

The parent(s) or legal guardian(s) of eligible patients were contacted via telephone. After full disclosure and discussion of the significance, procedures, risks, and benefits of the research protocol, verbal consent was obtained to complete the 3 neurodevelopmental survey tools prospectively. After enrollment, retrospective data collection was performed on all included patients. These collected clinical data included maternal and infant demographic data, mode of delivery, gestational age and birth weight, age at study, primary diagnosis, and nutrition data including total duration of PN, duration of IFER and mean lipid dose $(\mathrm{g} / \mathrm{kg} / \mathrm{d})$, and diagnosis of essential fatty acid deficiency. Essential fatty acid deficiency was defined as a triene to tetraene ratio of $>0.2$. Consistent with previous work done at our institution, a far more conservative triene to tetraene ratio of $>0.05$ was used as a criterion for picking up an early trend toward essential fatty acid deficiency (EFAD) but prior to any diagnosis of EFAD. Additional demographic data were obtained once verbal consent was documented, specifically data regarding number of hospital admissions, whether patients were receiving $\mathrm{PN}$ at the time of the study, general well-being, and socioeconomic variables. The socioeconomic status of enrolled patients was calculated using Hollingshead's Four
Factor Index of Social Status. ${ }^{37}$ The PEDS survey tool was completed during the initial phone call. The BASC-2 PRS-P and the ASQ-3 tools were mailed to consented patients and completed by the parent or legal guardian and then returned to study investigators. Study participants successfully completing all survey tools were provided with a monetary incentive in the form of a \$30 VISA gift card.

The primary outcome measure was the dichotomous score "at risk" vs "not at risk" based on 1 or more scales falling by $>2$ standard deviations below the mean on the ASQ-3. Secondary outcome measures included the trichotomous score of "low risk," "medium risk," or "high risk" on the PEDS tool as well as composite $t$ scores on the BASC-2 PRS-P tool.

\section{Survey Tools}

$A S Q-3 .^{38}$ The ASQ is the most widely used parent-reported developmental screening instrument. ${ }^{38}$ Concurrent validity ranges from $76 \%-88 \%$, and overall sensitivity and specificity are $75 \%$ and $86 \%$, respectively, compared with standardized measures. Interobserver reliability between parents and professional examiners is $94 \%$. The ASQ-3 consists of 19 age-specific questionnaires covering the age range of 4-60 months. A reading level of grades 4-6 ensures parental comprehension. The 30-item questionnaire takes 10-15 minutes to complete and covers 5 developmental domains: communication, gross motor, fine motor, problem solving, and personal social skills. Each domain is assessed by 6 questions answered with "yes," "sometimes," or "not yet," with a respective score of 10,5 , or 0 points.

Measurements from the ASQ-3 provide a developmental profile that evaluates global communication, gross motor, fine motor, problem-solving, and personal social skills domains. The ASQ-3 provides a score based on the child's scores in the 5 domains, which is compared with the age-standardized mean score. Referral for further assessment is advised when the score on any domain falls more than 2 standard deviations below the mean of the standardized sample. Scores are analyzed and interpreted as "above the cutoff," indicating the child's development appears to be on track; "close to the cutoff," indicating that additional learning activities are recommended and monitoring may be necessary; and "below the cutoff" (ie, 2 standard deviations below the mean), indicating that further assessment with a professional may be needed. For this analysis, scores were converted into a dichotomous outcome of "not at risk" or "at risk" as follows: patients scoring "above the cutoff" or "close to the cutoff" were deemed not at risk, while those patients scoring "below the cutoff" were termed at risk.

PEDS. ${ }^{39}$ The PEDS is a 10 -item parent report screening instrument for children ages birth to 8 years that identifies if children are at developmental risk, warranting further direct assessment. Items ask the parents if they have any concerns in 
specific developmental domains, including expressive language and articulation, receptive language, social-emotional, behavior, fine motor, gross motor, self-help, school, global/ cognitive, and other possible concerns. ${ }^{39}$ Parents respond with "no," "yes," or "a little" and are given the opportunity to comment in all assessed areas. The PEDS was standardized on a sample of 971 children. Compared with standardized measurements, sensitivity and specificity are $74 \%-80 \%$ and $70 \%-80 \%$, respectively.

Measurements from the PEDS provide a simple identification of patients at developmental risk based on the number of significant concerns identified. Patients with no concerns identified were termed "no concerns," those with $\geq 1$ non-significant concerns identified were categorized as "low risk," those with 1 significant concern were termed "medium risk," and those with $\geq 2$ significant concern were categorized as "high risk." ${ }^{40}$ For the purposes of this study and consistent with previously published work, patients were also dichotomized into 2 categories: at risk ( $\geq 1$ predictive/significant concern) and not at risk (no predictive/significant concerns). ${ }^{32,33}$

$B A S C-2$ PRS- $P .{ }^{35}$ The BASC-2 PRS-P is a validated 134-item questionnaire completed by parents/caregivers that assesses adaptive and problem behaviors of children between 2 and 5 years of age. ${ }^{35}$ The parent or caregiver rates the occurrence of various behaviors using a 4-point rating scale. Responses are scored and analyzed, yielding a profile that describes behavioral outcomes compared with normative values standardized across age. The normative population includes 500 children from 2-3 years old and 700 children from $4-5$ years old. The BASC-2 PRS-P takes 10-20 minutes to complete and requires a fourth-grade reading level. ${ }^{35}$ Limited construct validation data indicate strong correlations with similar scale scores, including significant correlations between BASC-2 externalizing, internalizing, and total score scales and similar scales; evidence of sensitivity to effects of teratogen exposure; and moderate to high correlations between parents' BASC-2 ratings. ${ }^{41-43}$

The score profile yields 4 composite scores: externalizing problems, internalizing problems, behavioral symptoms index, and adaptive skills. The composite scores are further broken down into 8 clinical scales and 4 adaptive scales. The clinical scales consist of the following: hyperactivity and aggression (externalizing problems), anxiety, depression and somatization (internalizing problems), atypicality, withdrawal, and attention problems (behavioral symptoms index). Adaptability, social skills, activities of daily living, and functional communication comprise the 4 adaptive scales.

Scores generated through analysis of the BASC-2 PRS-P are reported as $t$ scores with a mean score of 50 and a standard deviation of 10 . For clinical scales, higher scores indicate a higher risk and a greater likelihood of problems. The $t$ scores of $\geq 70$ indicate "clinically significant problems," $60-69$ is categorized as "at risk," and 20-59 indicates "average response" or typically developing. For adaptive scales, lower scores indicate a higher risk and a greater likelihood of problems. The $t$ scores of $\leq 30$ indicate "clinically significant problems," 31-40 is categorized as "at risk," and $>40$ indicates "average response" or typically developing. For the purposes of this study, scores were dichotomized to 2 categories: at risk ( $t$ scores $\geq 60$ for clinical scales and $\leq 40$ for adaptive scales) or not at risk $(t$ scores $<60$ for clinical scales and $>40$ for adaptive scales) for the 4 composite scores.

\section{Statistical Analysis}

All study participants were included in the statistical analysis. For those study participants who were unable to fully complete all 3 survey tools or who returned an incomplete set of survey tools, all available data were analyzed. All key data elements were recorded, and partial NDOs were evaluated. However, if the survey completion was missing the defined primary outcome measure, these study participants were not included in the final overall combined study analysis.

Data were analyzed using descriptive statistics, using the Student $t$ test for normally distributed variables and Wilcoxon rank sum tests for nonnormally distributed variables. The $\chi^{2}$ and Fisher exact tests were used for comparison of categorical variables. The relationship between NDOs and variables, including gestational age, sex, age at study, socioeconomic status, sepsis, number of operations/exposure to general anesthesia, trend toward EFAD (note: because a larger number of infants showed a slight increase in the triene to tetraene ratio $[>0.05]$, we retained this definition for those infants who were trending to a potential deficient state but had not yet reached it for statistical analyses), the total duration of PN, total duration of IFER, and the mean IVFE dose $(\mathrm{g} / \mathrm{kg} / \mathrm{d})$, was evaluated by performing a linear regression for continuous predictors and an independent samples $t$ test for categorical predictors on the BASC- $2 t$ scores. Logistic regression analyses were performed for dichotomized data. The $P$ value for the logistic regression models was assessed by using a likelihood ratio $\chi^{2}$ test. In addition, an exact logistic regression model was fitted for each predictor to verify the results. Bivariate correlation analysis was used to assess the association between the 3 neurodevelopmental survey tools. Data were analyzed using SAS software release 9.3 for Windows (SAS Institute, Cary, NC).

\section{Results}

A total of 62 patients meeting inclusion criteria were identified and contacted via telephone to participate in the study. Despite numerous attempts via telephone, 37 participants were unable to be contacted (nonconsented group). However, the remaining 25 families consented to complete the study. The PEDS was completed for all 25 consented patients (100\% response rate). The ASQ-3 and the BASC-2 PRS-P were successfully completed by 18 of the 25 consented patients. Thus, the completed 
Table 1. Demographic Data for Consented vs Nonconsented Groups.

\begin{tabular}{|c|c|c|c|}
\hline Characteristic & $\begin{array}{l}\text { Group Studied } \\
\text { (Consented) }(\mathrm{n}=25)\end{array}$ & $\begin{array}{c}\text { Group Not Studied } \\
\text { (Nonconsented) }(\mathrm{n}=37)\end{array}$ & $P$ Value \\
\hline Gestational age, mean \pm SD (range), wk & $34.8 \pm 4.2(25.86-40)$ & $32.6 \pm 4.2(25.71-38.57)$ & .05 \\
\hline Birth weight, mean $\pm \mathrm{SD}$ (range), $\mathrm{g}$ & $2376.9 \pm 929.9(650-3910)$ & $2013.8 \pm 797.7(700-3400)$ & .12 \\
\hline Age at study, mean $\pm \mathrm{SD}$ (range), mo & $53.8 \pm 11.8(35-81)$ & $54.2 \pm 11.7(33-77)$ & .88 \\
\hline Diagnosis, No. (\%) & & & .45 \\
\hline Hirschsprung & $2(8)$ & 0 & \\
\hline $\mathrm{CDH}$ & $7(28)$ & $4(10.8)$ & \\
\hline $\mathrm{EA} / \mathrm{TEF}$ & $2(8)$ & $2(5.4)$ & \\
\hline SBS & $5(20)$ & $9(24.3)$ & \\
\hline Gastroschisis & $6(24)$ & $13(34.1)$ & \\
\hline $\mathrm{NEC}$ & $2(8)$ & $4(10.8)$ & \\
\hline Intestinal perforation & 0 & $1(2.7)$ & \\
\hline Hydrops fetalis & 0 & $1(2.7)$ & \\
\hline Meconium ileus & $1(4)$ & 0 & \\
\hline Congenital cystic adenoid malformation & 0 & $1(2.7)$ & \\
\hline Atresia & 0 & $1(2.7)$ & \\
\hline Obstruction & 0 & $1(2.7)$ & \\
\hline Vaginal delivery, $\%$ & 54.6 & 37.8 & .21 \\
\hline Male sex, $\%$ & 80 & 23 & .13 \\
\hline Current $\mathrm{PN}$ at study, $\%$ & 8 & 2.3 & .56 \\
\hline Respiratory failure, $\%$ & 60 & 46 & .28 \\
\hline Hypoxia, \% & 4 & 24.3 & .04 \\
\hline Sepsis, $\%$ & 32 & 29.7 & .85 \\
\hline Retinopathy of prematurity, $\%$ & 8 & 13.5 & .69 \\
\hline Intraventricular hemorrhage, $\%$ & 4 & 8.1 & .64 \\
\hline Hypotension, \% & 44 & 46 & .88 \\
\hline $\begin{array}{l}\text { Number of operations, median ( } 25 \text { th percentile, } \\
75 \text { th percentile) }\end{array}$ & $7(5,14)$ & $4(2,7)$ & .01 \\
\hline $\begin{array}{l}\text { Duration of PN, median (25th percentile, } 75 \text { th } \\
\text { percentile), } d\end{array}$ & $67(39,100)$ & $61(36,94)$ & .52 \\
\hline $\begin{array}{l}\text { Duration lipid restriction, median ( } 25 \text { th percentile, } \\
75 \text { th percentile), d }\end{array}$ & $43(15,95)$ & $29(11,51)$ & .23 \\
\hline Dose of lipids, mean $\pm \mathrm{SD}$ (range), $\mathrm{g} / \mathrm{kg} / \mathrm{d}$ & $0.48 \pm 0.24(0.26-1.28)$ & $0.41 \pm 0.18(0.24-1)$ & .10 \\
\hline $\begin{array}{l}\text { Trend toward essential fatty acid deficiency } \\
\text { (triene to tetraene ratio }>0.05 \text { ), No. }\end{array}$ & 13 (of 16) & 10 (of 13) & $>.999$ \\
\hline $\begin{array}{l}\text { Essential fatty acid deficiency (triene to tetraene } \\
\text { ratio }>0.2 \text { ), No. }\end{array}$ & 1 (of 16) & 1 (of 13) & $>.999$ \\
\hline
\end{tabular}

$\mathrm{CDH}$, congenital diaphragmatic hernia; EA, esophageal atresia; EFA, essential fatty acid; NEC, necrotizing enterocolitis; PN, parenteral nutrition; SBS, short bowel syndrome; SD, standard deviation; TEF, tracheoesophageal fistula.

survey tool response rate for all 3 tools for the 25 consented patients was high, with an overall response rate of $72 \%$.

Demographic data for all 62 patients are displayed in Table 1. To assess whether the consented patients were representative of the entire cohort of patients previously treated with IFER, the consented group was compared with the nonconsented group. Overall, most demographics were not significantly different between these 2 populations; however, patients in the consented group differed in 2 categories. On average, infants were older at birth, with a mean gestational age in weeks (mean $\pm \mathrm{SD}$ [range]) of $34.8 \pm 4.2(25.86-40)$, compared with the nonconsented group, $32.6 \pm 4.2(25.71-38.57)(P=.05)$. There was also a greater incidence of postdelivery hypoxia, as documented in the medical record and/or diagnosis list, observed in the nonconsented group $(24.32 \%)$ compared with that in the consented group $(4 \%)(P=$ $.04)$. Gestational age and hypoxia may represent factors that affect neurodevelopmental outcomes irrespective of IFER. Therefore, the NDO results for the consented/studied group may be more reflective of the variable of interest: IFER.

\section{Results: ASQ-3}

Seventeen families completed the ASQ-3. The ASQ-3 domain scores were categorized as at risk vs not at risk (Table 2). Most 
Table 2. Dichotomized Results: Ages and Stages Questionnaires-3.

\begin{tabular}{lcc}
\hline Characteristic & At Risk, No. (\%) & Not at Risk, No. (\%) \\
\hline Communication & $1 / 18(5.6)$ & $17 / 18(94.4)$ \\
Gross motor & $3 / 17(17.7)$ & $14 / 17(82.4)$ \\
Fine motor & $1 / 17(5.9)$ & $16 / 17(94.1)$ \\
Problem solving & $1 / 18(5.6)$ & $17 / 18(94.4)$ \\
Personal social & $2 / 17(11.8)$ & $15 / 17(88.2)$ \\
\hline
\end{tabular}

of the study participants scored "not at risk" for all 5 domains of communication, gross motor, fine motor, problem solving, and personal social. Three of 17 patients $(17.7 \%)$ scored "at risk" in the domain of gross motor. In this domain, betweengroup differences were identified. Specifically, mean gestational age in weeks (mean $\pm \mathrm{SD}$ [range]) was $31.5 \pm 2$ (29.7-33.7) for the patients at risk vs was $35.9 \pm 2.7(30-39.1)$ for the group not at risk $(P=.02)$. In addition, the mean age of those in the at-risk group was $52 \pm 18.3(36-72)$ months at the time of the study vs $55.3 \pm 12.8(35-81)$ months in the group not at risk, but the group difference was not statistically significant. The median duration of IFER was 53 days (25th, 75th percentile: 5,95 days) vs 44.5 days (25th, 75 th percentile: 12 , 113 days), and the mean lipid dose $(\mathrm{g} / \mathrm{kg} / \mathrm{d})$ was $0.46 \mathrm{~g} \pm 0.18$ $(0.32-0.65)$ vs $0.54 \pm 0.29(0.26-1.28)$ in the groups at risk and not at risk, respectively. Differences between IFER duration and mean lipid dose were not statistically significantly different between the groups at risk and not at risk.

To examine the risk factors contributing to at-risk NDOs, we performed logistic regression analyses (Table 3 ) for all 5 domains. In the areas of communication, fine motor, and problem solving, no variables were statistically significant predictors of outcomes. Gestational age was an independent predictor in the domains of gross motor $(P=.03)$ and personal social $(P$ $=.04)$, with patients at a younger gestational age at higher risk. An inspection of 3 specific IFER-related covariates failed to show significance; specifically, IFER duration, mean IVFE dose, and development of EFAD were not significant predictors of at-risk ASQ-3 outcomes.

\section{Results: PEDS}

A total of 25 families completed the PEDS survey. Eleven study participants $(44 \%)$ had no concerns identified and were deemed to be at no risk. Six patients $(24 \%)$ had $\geq 1$ nonsignificant concern or 1 significant concern, and these latter 6 children were categorized as low risk and medium risk, respectively. Only 2 patients $(8 \%)$ were at high risk with $\geq 2$ significant concerns identified (Figure 1). The data were dichotomized, such that study patients with $>1$ significant concern were categorized as at risk and those with no significant concerns were categorized as not at risk. A greater proportion of patients were found to be not at risk $(\mathrm{n}=17[68 \%])$ vs only 8 patients found to be at risk (32\%). Logistic regression analyses were performed to examine predictors of risk categorization (Table 3). The only variable found to be a significant predictor of at-risk outcome was an older average age at study enrollment $(P<$ $.01)$. No other variables, including IFER duration, mean IVFE dose, or development of EFAD, were statistically significant predictors of at-risk PEDS outcomes.

\section{Results: BASC-2 PRS-P}

A total of 18 subject families completed the BASC-2 PRS-P assessment (Table 4). Mean $t$ scores for all 4 composite domains fell within the average range of 41-59, indicating that the study sample generally scored within the normative response range. Mean $t$ scores for 7 of 8 clinical scales and all 4 adaptive scales also fell within the range of 41-59. Mean $t$ scores on the clinical scale of somatization fell outside the average range, $60.5 \pm 13.65$ (range, 43-88).

Descriptive statistics for the study population falling within the 3 categories of "typically developing," "at risk," or "clinically significant problems" were computed. "Typically developing" categorization was observed in $89 \%$ of patients for externalizing problems, $67 \%$ for internalizing problems, and $83 \%$ for behavioral symptoms index and adaptive skills. All clinical and adaptive scales had $>50 \%$ of study participants scoring "typically developing," with the exception of somatization, in which $55.5 \%$ of participants fell within the "at-risk" or "clinically significant problems" category.

Linear regression modeling was performed to examine predictors of BASC-2 PRS-P $t$ scores in the 4 composite scales. No variables were independent predictors of internalizing or behavioral problems scores. Gestational age emerged as an independent predictor of adaptive skills, $P=.03\left(r^{2}=0.26\right)$, with lower gestational age associated with poor scores in this domain. Marginal significance was also seen in the area of behavioral problems, $P=.08\left(r^{2}=0.18\right)$, for gestational age. Average IVFE dose $(\mathrm{g} / \mathrm{kg} / \mathrm{d})$ was a significant predictor of externalizing $\left(r^{2}=0.26, P=.03\right)$ for those patients receiving higher average daily IVFE dose. The $t$ scores were $\geq 70$ when the mean IVFE dose was $>1.93 \mathrm{~g} / \mathrm{kg} / \mathrm{d}$. The association between average IVFE dose and internalizing problems was marginally significant $\left(r^{2}=0.17, P=.09\right)$. Thus, administration of higher doses of IVFE was associated with some worsening of behavioral problems; however, no adverse behavioral scores were detected in those infants receiving restricted doses of IVFE. However, when the Bonferroni correction was applied, statistical significance was no longer observed for any of the variables analyzed.

Dichotomized data are illustrated in Figure 2. Most study patients were observed to be not at risk in the composite scales, with $16(88.9 \%)$ not at risk in externalizing problems, 12 $(66.7 \%)$ in internalizing problems, and $15(83.3 \%)$ in behavioral problems and adaptive skills. Logistic regression analysis revealed that a younger gestational age was marginally 
Table 3. Logistic Regression Analyses of Key Predictive Variables on NDO Measurements.

\begin{tabular}{lcccc}
\hline Variable & Test & Domain & Odds Ratio & $95 \%$ Confidence Interval \\
\hline Gestational age & ASQ-3 & Gross motor & 0.64 & $0.33-0.97$ \\
& ASQ-3 & Personal social & 0.58 & $0.16-0.97$ \\
Older age at study & PEDS & & 1.12 & $1.03-1.26$ \\
Higher average dose of IVFE & BASC-2 PRS-P & Internalizing problems & 45.2 & $1.06-36,680.5$ \\
\hline
\end{tabular}

ASQ-3, Ages and Stages Questionnaires-3; BASC-2 PRS-P, Behavior Assessment System for Children, Second Edition Preschool, Parent; IVFE, intravenous fat emulsion; PEDS, Parents' Evaluations of Developmental Status.

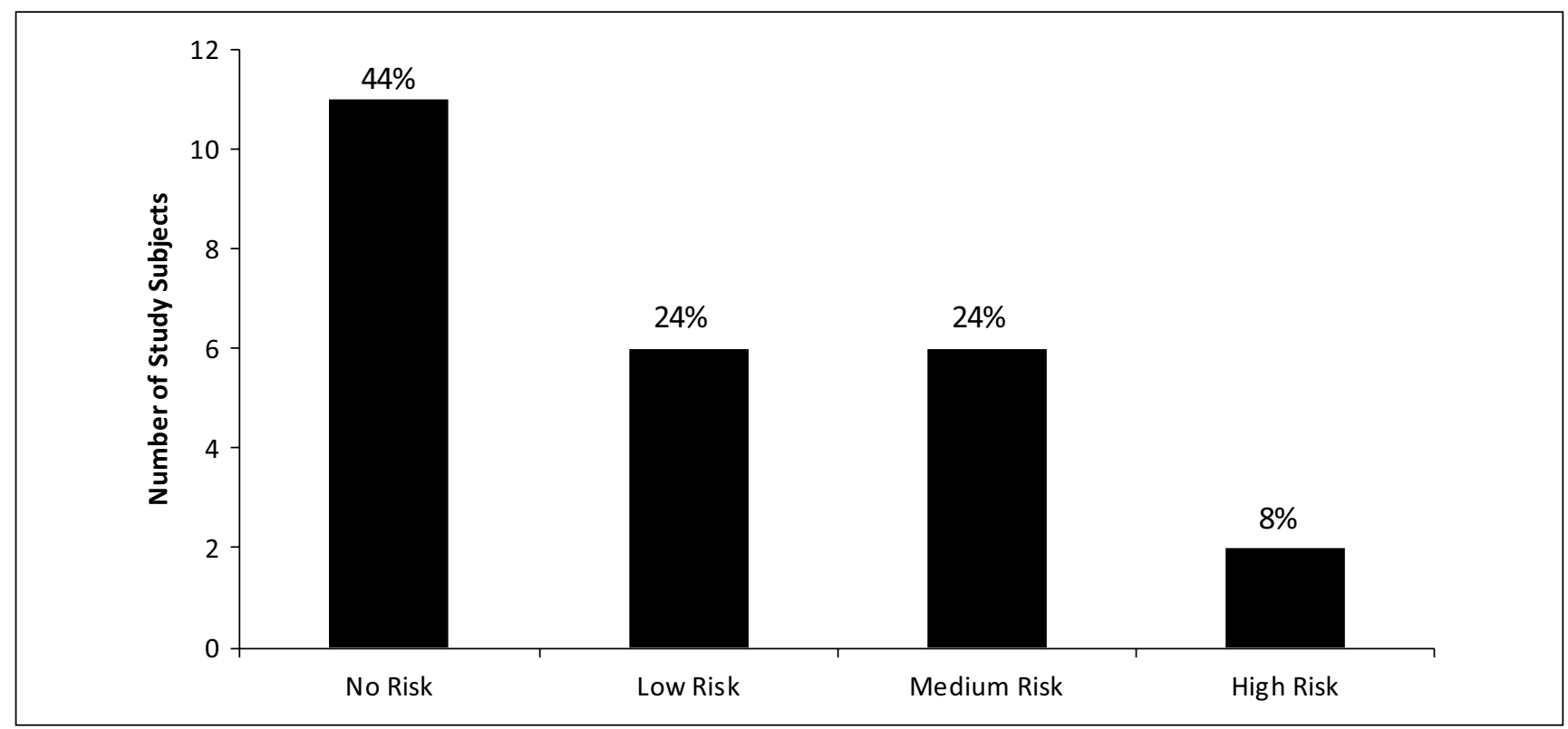

Figure 1. Graphical description of overall results on the Parents' Evaluations of Developmental Status tool for all 25 consented study subjects. Forty-four percent $(n=11)$ of patients had no concerns identified (no risk), $24 \%(n=6)$ had $\geq 1$ nonsignificant concern (low risk) or 1 significant concern (medium risk), and $8 \%(n=2)$ had $\geq 2$ significant concerns and were categorized as high risk for developmental concerns.

predictive of lower adaptive skills $(P=.08)$. Furthermore, a higher mean IVFE dose $(\mathrm{g} / \mathrm{kg} / \mathrm{d})$ was an independent predictor of risk for the domain of internalizing problems $(P=.05)$, and higher IVFE dose also showed a marginal significance for externalizing problems $(P=.10)$. However, no other variables were statistically significant predictors of outcome in any of the 4 composite scales when data were dichotomized (Table 3 ).

\section{Secondary Analysis}

The agreement between and relative sensitivity of the ASQ-3 and PEDS surveys is displayed in Table 5. Only 1 of the 18 patients with complete data from both surveys was found to be at risk on both the ASQ-3 and the PEDS survey tools, indicating that the 2 tools identified different at-risk patients. As such, for the purposes of this secondary analysis, an overall risk categorization was assigned to each of the 18 patients with complete data sets if they were at risk on either the PEDS or the
ASQ-3 tool. When dichotomized and analyzed in this way, most patients overall were not at risk $(n=11[61.1 \%])$ vs those observed to be at risk $(n=7$ [38.9\%]). Logistical regression analysis revealed that no variables were independent predictors of overall risk categorization (Table 6). In particular, neither restricted IVFE dose nor duration of IFER was associated with being at risk. Between-group differences are described in Table 7 for the groups overall that were not at risk vs at risk. There were no significant differences in characteristics between the 2 groups. Finally, the influence of overall risk categorization on observed outcomes on the BASC-2 PRS-P is described. For those patients at risk in the overall combined risk categorization, scores on the BASC-2 PRS-P for behavioral symptom index and adaptive skills were predicted to be at risk $(P=.04)$.

Post hoc statistical analysis. A limited number of patients were included in this study due to difficulty in successfully contacting and consenting patients and the 
Table 4. Results: BASC-2 PRS-P ( $\mathrm{n}=18)$.

\begin{tabular}{|c|c|c|c|c|}
\hline Characteristic & Score, Mean \pm SD (range) & Typically Developing & At Risk & Clinically Significant Problems \\
\hline Externalizing problems & $49.6 \pm 7.5(37-64)$ & $16(88.9)$ & $2(11.1)$ & 0 \\
\hline Hyperactivity & $52 \pm 8.3(38-74)$ & $15(83.3)$ & $2(11.1)$ & $1(5.6)$ \\
\hline Aggression & $47.3 \pm 7.2(36-59)$ & $18(100)$ & 0 & 0 \\
\hline Internalizing problems & $54.5 \pm 2.6(37-75)$ & $12(66.7)$ & $4(22.2)$ & $2(11.1)$ \\
\hline Anxiety & $49.5 \pm 8.6(36-66)$ & $17(94.4)$ & $1(5.6)$ & 0 \\
\hline Depression & $50.1 \pm 9.4(32-71)$ & $16(88.9)$ & $1(5.6)$ & $1(5.6)$ \\
\hline Somatization & $60.5 \pm 13.7(43-88)$ & $8(44.4)$ & $6(33.3)$ & $4(22.2)$ \\
\hline Behavioral symptom index & $51.6 \pm 10.2(36-77)$ & $15(83.3)$ & $2(11.1)$ & $1(5.56)$ \\
\hline Atypicality & $53.2 \pm 13.1(39-81)$ & $12(66.7)$ & $4(22.2)$ & $2(11.1)$ \\
\hline Withdrawal & $52 \pm 11.4(30-78)$ & $14(77.8)$ & $3(16.7)$ & $1(5.6)$ \\
\hline Attention problems & $52.7 \pm 10.5(38-71)$ & $14(77.8)$ & $3(16.7)$ & $2(11.1)$ \\
\hline Adaptive skills & $50.8 \pm 11.5(23-70)$ & $15(83.3)$ & $2(11.1)$ & $1(5.56)$ \\
\hline Adaptability & $51.1 \pm 12.1(32-72)$ & $16(88.9)$ & $2(11.1)$ & 0 \\
\hline Social skills & $53.8 \pm 11.6(33-70)$ & $15(83.3)$ & $3(16.7)$ & 0 \\
\hline Activities of daily living & $48.2 \pm 9.6(31-61)$ & $15(83.3)$ & $4(22.2)$ & 0 \\
\hline Functional communication & $48.8 \pm 10.7(19-71)$ & $16(88.9)$ & $1(5.6)$ & $1(5.6)$ \\
\hline
\end{tabular}

Overall results, reported as mean $t$ scores \pm SD (range) for the Behavior Assessment System for Children, Second Edition Preschool, Parent (BASC-2 PRS-P) are displayed for the 18 study participants who completed the BASC-2 PRS-P. The 4 composite scores are broken down into 8 clinical scales and 4 adaptive scales: (1) externalizing problems (hyperactivity, aggression), (2) internalizing problems (anxiety, depression, somatization), (3) behavioral symptom index (atypicality, withdrawal, attention problems), and (4) adaptive skills (adaptability, social skills, activity of daily living, functional communication). Higher scores on clinical scales and lower scores on adaptive scales indicate a higher risk and greater likelihood of problems. Results for participants with scores categorized as "typically developing," "at risk," and "clinically significant problems" are reported as total number within each group and percentage of participants.

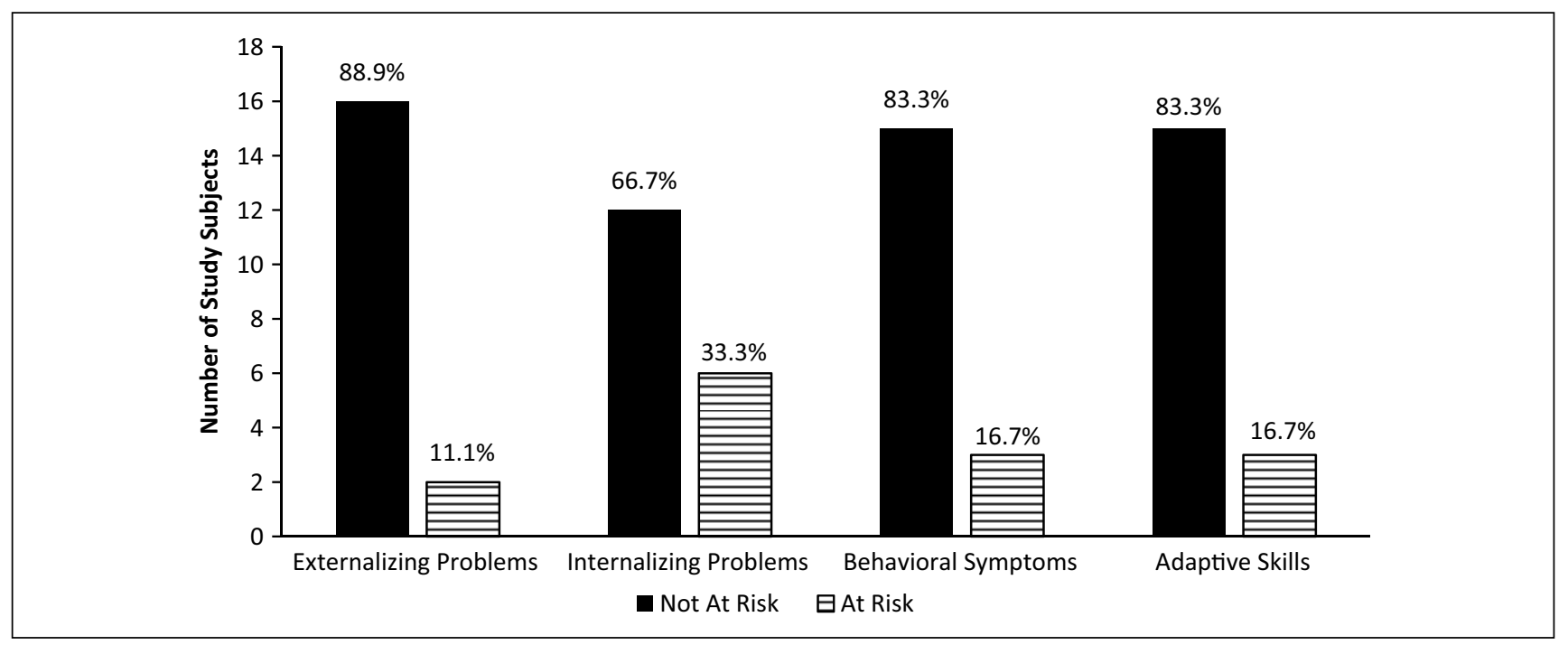

Figure 2. Behavior Assessment System for Children, Second Edition Preschool, Parent (BASC-2 PRS-P) risk categorization: study participants falling into the not at risk vs at-risk category is graphically displayed for the 4 composite domains.

unavailability of complete survey data for some consented patients. As such, while some patients were identified to be at risk for poor neurodevelopmental outcomes, the true, absolute risk cannot be conclusively determined from these data. Therefore, a post hoc sample size calculation cannot be accurately determined from these pilot data.
Similarly, the ability to stratify results by several covariates, such as exposure to enteral nutrition (EN), PN duration, IFER duration, gestational age, and development of EFAD, would have been of interest and may have provided valuable information. However, due to the small sample size, this type of statistical analysis was not possible but should be considered for larger studies aimed at further evaluating NDOs associated with IFER. 
Table 5. ASQ-3/PEDS Result Agreement.

\begin{tabular}{lcl}
\hline & \multicolumn{2}{c}{ PEDS, No. (\%) } \\
\cline { 2 - 3 } ASQ-3 & Not at Risk & At Risk \\
\hline Not at risk & $11(61.1)$ & $3(16.7)$ \\
At risk & $3(16.7)$ & $1(5.6)$ \\
\hline
\end{tabular}

Tabular description of agreement between the Ages and Stages Questionnaires-3 (ASQ-3) and Parents' Evaluations of Developmental Status (PEDS) survey tools for the 18 subjects completing both surveys. One patient was at risk on both the ASQ-3 and PEDS survey tools.

\section{Discussion}

A recognized approach to the management of PNAC is the reduction of soybean-based IVFE. While biochemical reduction in liver function tests and potential benefits to the liver have been shown with this approach, ${ }^{16,17,19,20}$ long-term outcomes - specifically, neurodevelopmental and behavioral outcomes - have not been described previously. To our knowledge, this is the first published report of neurodevelopmental and behavioral outcomes in pediatric patients previously treated with IFER. Importantly, most patients were found to be not at risk, and in those with negative NDOs, regression analysis showed that IFER-related variables were not predictive of neurodevelopmental or behavioral outcomes.

The essential fatty acids are known to be important for neurodevelopment. Specifically, docosahexaenoic acid (DHA), arachidonic acid (AA), and long-chain polyunsaturated fatty acids are important for neural development and visual development within the first year of life. ${ }^{21}$ The essential fatty acids, linoleic and $\alpha$-linolenic acids, are converted to AA and DHA and are typically provided through EN (eg, breast milk or enteral formulas). However, for PN-dependent patients, the essential fatty acids must be provided through IVFE. The delicate balance between providing these essential nutrients during infancy, a critical period of growth and development, and preventing the development of PNAC through alterations in the provision of IVFE has raised the question of long-term neurodevelopmental effects associated with these methods. To date, little has been known regarding the effects of reducing IVFE, including the types of neurodevelopmental domains of concern and the influence of IFER on outcomes. Of the IFER-related variables analyzed, IVFE dose was the only variable that was associated with outcomes. Interestingly, however, higher lipid doses, to our surprise, were associated with worse NDO outcomes in the domain of externalizing problems on the BASC-2 PRS-P. Although this outcome may be due to chance, there are 2 likely explanations for this finding. First, it is possible that those patients treated with higher doses of lipids are at greater risk for poor NDOs. The optimal dose and source of IVFE are a topic of current investigation, and the effects of exogenous supplementation via IVFE or the enteral route on the developing brain have not been elucidated conclusively. ${ }^{44}$ Second, it may be that the higher dose of lipids is reflective of those patients who received IFER for a longer duration and underwent dose titration either due to suboptimal growth or the development of EFAD. Due to the nature of the retrospective data collection, the specific clinical indications for IVFE dose escalation were unable to be obtained; however, escalation was chosen for either failure to demonstrate adequate weight gain or with any evidence of a trend toward EFAD. Thus, the interpretation of these data is challenging, specifically with respect to EFAD. One limitation to the interpretation of the EFAD data is that there was a significant amount of missing data. Specifically, several patients did not have an essential fatty acid profile drawn, and thus the numbers reported represent those patients in each group who had an essential fatty acid profile drawn and were found to be deficient. Still, the development of EFAD was not found to be a predictor of poor neurodevelopmental outcomes.

$\mathrm{EN}$, both breast milk as well as enteral formula, provides an important source of essential fatty acids. While stratification of neurodevelopmental results by this covariate was not possible due to small sample size, at the completion of the study, we performed a retrospective analysis of exposure to EN. A description of the average percent enteral intake and parenteral intake at the time of IFER initiation and for the first 6 weeks of IFER is provided in Table 8. Evaluation over the first 6 weeks was chosen to account for the most critical period of brain development. An additional rationale was that this comprised the period when the greatest number of infants was on IFER. Overall, for the first 6 weeks of IFER, the mean percent of EN ranged from $11.7 \%-25.3 \%$, and the mean percent of $\mathrm{PN}$ ranged from $71.1 \%-89.5 \%$ for the 18 patients completing all 3 NDO surveys. While EN comprised a small proportion of the calories over the first 6 weeks of IFER, most calories were provided from PN. Furthermore, cautious interpretation of enteral intake and exposure to enteral essential fatty acids is recommended, since patients dependent on long-term PN may have variable absorption of EN, and exposure to EN may not accurately reflect the absorption of nutrients. We believe that the results of this study and the influence of IFER on NDOs can be interpreted and attributed to exposure to IFER rather than enteral intake. However, due to the retrospective nature of this study, future studies are warranted, which may be designed to prospectively evaluate the effects of enteral and parenteral provision of essential fatty acids and to stratify results based on exposure to EN.

The neurodevelopmental screening tools used in this investigation were parental surveys rather than direct assessments of development, behavior, and neuropsychological status. While these survey tools are validated measures,,$^{35,38-40}$ they offer gross measurements of risk and are not designed to assess subtle developmental deviations, including abnormalities in attention and early executive dysfunction. Despite this limitation, the study measurement is strengthened by the combination of assessment tools. Instruments are sensitive to different aspects 
Table 6. Logistic Regression Analyses of IFER-Related Variables on Overall Risk Categorization.

\begin{tabular}{lccc}
\hline Variable & Odds Ratio & 95\% Confidence Interval & $P$ Value \\
\hline IFER duration & 1 & $0.99-1.02$ & .54 \\
IVFE dose & 9.97 & $0.37-1352.89$ & $0-2.2$ \\
Trend toward essential fatty acid deficiency & 0.12 & & .18 \\
$\quad$ triene to tetraene ratio $>0.05)$ & & \\
\hline
\end{tabular}

IFER, intravenous fat emulsion reduction; IVFE, intravenous fat emulsion.

Table 7. Characteristics and BASC-2 PRS-P Risk Categorization Overall for Patients Not at Risk vs At Risk.

\begin{tabular}{|c|c|c|c|}
\hline Characteristic & Not at Risk $(\mathrm{n}=11)$ & At Risk $(\mathrm{n}=7)$ & $P$ Value \\
\hline Gestational age, mean \pm SD (range), wk & $36.4 \pm 2.6(31.4-40)$ & $33.8 \pm 3.7(29.7-39.1)$ & .09 \\
\hline Birth weight, mean $\pm \mathrm{SD}$ (range), $\mathrm{g}$ & $2594.9 \pm 589(1375-3353)$ & $2037.1 \pm 939.9(972-3500)$ & .15 \\
\hline Age at study, mean $\pm \mathrm{SD}$ (range), mo & $51.1 \pm 10.3(35-72)$ & $59.3 \pm 16.1(36-81)$ & .2 \\
\hline Socioeconomic status score, mean \pm SD (range) & $39.2 \pm 15.3(22-63.5)$ & $26.9 \pm 9.3(9-39.5)$ & .29 \\
\hline Diagnosis, No. & & & .7 \\
\hline Hirschsprung & 2 & 0 & \\
\hline $\mathrm{CDH}$ & 2 & 1 & \\
\hline $\mathrm{EA} / \mathrm{TEF}$ & 1 & 1 & \\
\hline SBS & 1 & 1 & \\
\hline Gastroschisis & 4 & 2 & \\
\hline NEC & 0 & 2 & \\
\hline Intestinal perforation & 0 & 0 & \\
\hline Hydrops fetalis & 0 & 0 & \\
\hline Meconium ileus & 1 & 0 & \\
\hline Congenital cystic adenoid malformation & 0 & 0 & \\
\hline Atresia & 0 & 0 & \\
\hline Obstruction & 0 & 0 & \\
\hline Vaginal delivery, $\%$ & 70 & 50 & .61 \\
\hline Male sex, $\%$ & 81.8 & 71.4 & $>.999$ \\
\hline Current PN at study, \% & 0 & 28.6 & .14 \\
\hline Respiratory failure, $\%$ & 45.5 & 71.4 & .37 \\
\hline Нypoxia, \% & 0 & 0 & \\
\hline Sepsis, $\%$ & 36.4 & 42.9 & $>.999$ \\
\hline Retinopathy of prematurity, $\%$ & 0 & 0 & \\
\hline Intraventricular hemorrhage, $\%$ & 0 & 0 & \\
\hline Hypotension, \% & 27.3 & 28.6 & $>.999$ \\
\hline $\begin{array}{l}\text { Number of operations, median ( } 25 \text { th percentile, } \\
75 \text { th percentile) }\end{array}$ & $7(4,11)$ & $7(5,17)$ & .7 \\
\hline $\begin{array}{l}\text { Duration of } \mathrm{PN} \text {, median ( } 25 \text { th percentile, } 75 \text { th } \\
\text { percentile), } \mathrm{d}\end{array}$ & $65(23,120)$ & $79(53,100)$ & .48 \\
\hline $\begin{array}{l}\text { Duration lipid restriction, median ( } 25 \text { th percentile, } \\
75 \text { th percentile), d }\end{array}$ & $20(5,113)$ & $65(9,95)$ & .49 \\
\hline Dose of lipids, mean $\pm \mathrm{SD}$ (range), $\mathrm{g} / \mathrm{kg} / \mathrm{d}$ & $0.44 \pm 0.19(0.26-1)$ & $0.62 \pm 0.33(0.32-1.28)$ & .26 \\
\hline $\begin{array}{l}\text { Trend toward essential fatty acid deficiency } \\
\text { (triene to tetraene ratio }>0.05 \text { ), No. }\end{array}$ & 4 (of 5) & 3 (of 5) & .44 \\
\hline $\begin{array}{l}\text { Severe essential fatty acid deficiency (triene to } \\
\text { tetraene ratio }>0.2 \text { ), No. }\end{array}$ & 0 (of 5) & 1 (of 5) & $>.999$ \\
\hline \multicolumn{4}{|l|}{ BASC-2 PRS-P risk categorization, $\%$} \\
\hline Externalizing problems & 0 & 28.6 & .14 \\
\hline Internalizing problems & 27.3 & 42.9 & .63 \\
\hline Behavioral symptom index & 0 & 42.9 & .04 \\
\hline Adaptive skills & 0 & 42.0 & .04 \\
\hline
\end{tabular}

BASC-2 PRS-P, Behavior Assessment System for Children, Second Edition Preschool, Parent; CDH, congenital diaphragmatic hernia; EA, esophageal atresia; EFA, essential fatty acid; NEC, necrotizing enterocolitis; PN, parenteral nutrition; SBS, short bowel syndrome; SD, standard deviation; TEF, tracheoesophageal fistula. 
Table 8. Mean Percent Enteral Nutrition vs Parenteral Nutrition During the First 6 Weeks of IFER.

\begin{tabular}{lcc}
\hline Characteristic & Enteral Nutrition, Mean \pm SD (Range), $\%$ & Parenteral Nutrition, Mean \pm SD (Range), \% \\
\hline At time of IFER & $11.7 \pm 17.8(0-60)$ & $89.5 \pm 17.2(40-100)$ \\
Week 1 of IFER & $23.4 \pm 29.5(0-100)$ & $75.6 \pm 28.2(0-100)$ \\
Week 2 of IFER & $21.7 \pm 25.2(0-75)$ & $74.8 \pm 25.4(25-100)$ \\
Week 3 of IFER & $25.3 \pm 29.2(0-90)$ & $71.1 \pm 28.4(10-100)$ \\
Week 4 of IFER & $18 \pm 23.8(0-50)$ & $77.1 \pm 24.8(50-100)$ \\
Week 5 of IFER & $19.8 \pm 23.1(0-50)$ & $75.5 \pm 23.9(50-100)$ \\
Week 6 of IFER & $18.1 \pm 22.6(0-50)$ & $81.9 \pm 22.6(50-100)$ \\
\hline
\end{tabular}

IFER, intravenous fat emulsion reduction.

of risk. This was seen in our study findings, with differing areas of concern identified on the various tools: gross motor ( $17.6 \%$ of children evaluated) on the ASQ-3 and somatization (22.2\% of children evaluated) on the BASC-2 PRS-P. Furthermore, consistent with previous research, the ASQ-3 and PEDS identified differences in those considered at risk. ${ }^{33}$ As noted by Sices et $\mathrm{al}^{33}$ these instruments use different formats, including assessment of concerns vs assessment of skills, as well as different approaches to cutoff for risk that may affect sensitivity. In addition, the PEDS is validated across an older age range, which may explain some of the differences, since this tool may be more sensitive in picking up outcomes once peer relationships are known and children are at school age, whereas the ASQ-3 may be more sensitive to risk in younger children. Because the correlation between the PEDS and ASQ-3 was not strong in our study, we performed the secondary analysis, which combined the data to include patients who were deemed at risk on either the PEDS tool or the ASQ-3 tool. In doing so, we found that $61.1 \%$ (11 of 18 children) overall were not at risk in our study, whereas $38.9 \%$ ( 7 of 18 children) were deemed at risk. A comparison of the 2 groups revealed that there were no significant demographic or clinical differences between those at risk and those not at risk. Importantly, IFER-related variables were not found to be significant predictors of outcome.

Several risk factors for negative neurodevelopmental and behavior outcomes for neonates have previously been investigated and described in the literature, including, but not limited to, prematurity, exposure to certain medications, hypoxia at time of birth, development of intraventricular hemorrhage, chronic lung disease, episodes of sepsis, and retinopathy of prematurity. ${ }^{45-50}$ The relatively strict inclusion and exclusion criteria of our study allowed for the influence of confounding variables to be limited and for the variables of interestnamely, IFER-related variables - to be isolated. Likewise, of the identified participants who consented to the study, there were fewer episodes of hypoxia, and the consented group had an older gestational age at the time of birth. While accounting for confounding variables allowed for a clean data analysis, a potential limitation is that the sample size was small, thus potentially limiting the power to detect significant differences as well as the generalizability of the findings. Furthermore, additional variables such as exposure to EN, suboptimal energy delivery, and normal growth assessments may affect NDOs. Although adverse neurodevelopmental outcomes may come from poor nutrition delivery, we carefully followed our infants twice weekly to ensure adequate growth. Our infants had increased soybean-based IVFE if growth fell below accepted means. Furthermore, using even more stringent IVFE restriction, Cober et $\mathrm{al}^{16,17}$ demonstrated normal weight and linear length via standard growth curves and World Health Organization $z$ scores. While these were not specifically evaluated in this study, we believe that the results are not significantly limited based on previous findings.

Of importance, early gestational age and low birth weight have been linked to long-term neurodevelopmental delays. ${ }^{51-53}$ In a recent study that evaluated 2.5-year neurodevelopmental outcomes associated with prematurity, $31 \%$ were found to have mild disability, $16 \%$ with moderate disability, and $11 \%$ with severe disability, with overall rates of moderate and severe disability decreasing with increasing gestational age. ${ }^{52}$ It is, therefore, not surprising that in our investigation, gestational age was identified as a predictive factor for negative NDOs. The surgical population may also represent a patient population that is at a higher baseline risk for negative NDOs, as repeated exposure to anesthetics has been linked to developmental problems such as learning disabilities. ${ }^{54,55}$ In our study, while between-group differences did not exist, the median number of operations in the groups at risk and not at risk was seven. Thus, it is probable that while we did identify a portion of our cohort to be at risk, they are representative of a higher risk group at baseline and would have been at risk even if they had not been treated with IFER. It must also be noted that the neurodevelopmental outcomes of the children included in our study were compared with population norms rather than to a sample with similar neonatal and surgical baseline characteristics. Future investigations will need to focus on prospective evaluations that compare a larger sample of children treated with IFER with those who receive standard IVFE therapy who are comparable at baseline to more definitively assess the influence of IFER on neurodevelopmental outcomes. Comparisons of IFER-treated children compared with those who receive alternative fat emulsions (ie, 
fish oil, olive oil, or combination fat emulsions) are also important future directions. In addition, a larger study sample would allow for the combined influence of covariates to be analyzed, potentially elucidating the additive effect of factors such as gestational age and IFER-related variables. Finally, we did not specifically collect data pertaining to early intervention programs. In general practice, our neonatal intensive care unit does screen patients, and all premature neonates are screened at 1 year of life. Additional referrals to early intervention were done on an ad hoc basis by the primary care physicians or surgeons. Future studies should incorporate this into the study design to address this limitation.

\section{Conclusion}

This study represents the first report of NDOs in pediatric patients treated with IFER. The neurodevelopmental outcomes of infants previously treated with IFER were within the normative range most of the time in the population evaluated in this study. Although roughly one-third of the patients were at risk on at least 1 measure, we were unable to demonstrate any causal relation of these at-risk infants to the use of IFER. These preliminary results do not reveal significant associations with the variables related to IFER such as IFER duration, IVFE dose, and the development of EFAD, since these were not predictive of poor neurodevelopmental outcomes. However, several limitations to this study exist, including small sample size, retrospective data collection, and parental report of neurodevelopmental outcomes. Thus, further research is needed to conclusively determine the influence of IFER on long-term neurodevelopmental outcomes, which may be accomplished, ideally, through a large, multisite, randomized, prospective study.

\section{References}

1. Kelly DA. Liver complications of pediatric parenteral nutrition-epidemiology. Nutrition. 1998;14:153-157.

2. Kelly DA. Intestinal failure-associated liver disease: what do we know today? Gastroenterology. 2006;130:S70-S77.

3. Carter BA, Karpen SJ. Intestinal failure-associated liver disease: management and treatment strategies past, present, and future. Semin Liver Dis. 2007;27:251-258.

4. Ginn-Pease ME, Pantalos D, King DR. TPN-associated hyperbilirubinemia: a common problem in newborn surgical patients. $J$ Pediatr Surg. 1985;20:436-439.

5. Spencer AU, Neaga A, West B, et al. Pediatric short bowel syndrome: redefining predictors of success. Ann Surg. 2005;242:403-412.

6. Koseesirikul P, Chotinaruemol S, Ukarapol N. Incidence and risk factors of parenteral nutrition-associated liver disease in newborn infants. Pediatr Int. 2012;54:434-436.

7. Drongowski RA, Coran AG. An analysis of factors contributing to the development of total parenteral nutrition-induced cholestasis. JPEN J Parenter Enteral Nutr. 1989;13:586-589.

8. Carter BA, Shulman RJ. Mechanisms of disease: update on the molecular etiology and fundamentals of parenteral nutrition associated cholestasis. Nat Clin Pract Gastroenterol Hepatol. 2007;4:277-287.

9. Moss RL, Amii LA. New approaches to understanding the etiology and treatment of total parenteral nutrition-associated cholestasis. Semin Pediatr Surg. 1999;8:140-147.
10. Kumpf VJ. Parenteral nutrition-associated liver disease in adult and pediatric patients. Nutr Clin Pract. 2006;21:279-290.

11. Rangel SJ, Calkins CM, Cowles RA, et al. Parenteral nutrition-associated cholestasis: an American Pediatric Surgical Association Outcomes and Clinical Trials Committee systematic review. J Pediatr Surg. 2012;47:225-240.

12. Diamond IR, Sterescu A, Pencharz PB, Kim JH, Wales PW. Changing the paradigm: Omegaven for the treatment of liver failure in pediatric short bowel syndrome. J Pediatr Gastroenterol Nutr. 2009;48:209-215.

13. Gura KM, Lee S, Valim C, et al. Safety and efficacy of a fish-oil-based fat emulsion in the treatment of parenteral nutrition-associated liver disease. Pediatrics. 2008;121:e678-e686.

14. Allardyce DB. Cholestasis caused by lipid emulsions. Surg Gynecol Obstet. 1982;154:641-647.

15. Colomb V, Jobert-Giraud A, Lacaille F, Goulet O, Fournet JC, Ricour C. Role of lipid emulsions in cholestasis associated with long-term parenteral nutrition in children. JPEN J Parenter Enteral Nutr. 2000;24:345-350.

16. Cober MP, Killu G, Brattain A, Welch KB, Kunisaki SM, Teitelbaum DH. Intravenous fat emulsions reduction for patients with parenteral nutritionassociated liver disease. J Pediatr. 2012;160:421-427.

17. Cober MP, Teitelbaum DH. Prevention of parenteral nutrition-associated liver disease: lipid minimization. Curr Opin Organ Transplant. 2010;15:330-333.

18. Nehra D, Fallon EM, Carlson SJ, et al. Provision of a soy-based intravenous lipid emulsion at $1 \mathrm{~g} / \mathrm{kg} / \mathrm{d}$ does not prevent cholestasis in neonates. JPEN J Parenter Enteral Nutr. 2013;37:498-505.

19. Sanchez SE, Braun LP, Mercer LD, Sherrill M, Stevens J, Javid PJ. The effect of lipid restriction on the prevention of parenteral nutrition-associated cholestasis in surgical infants. J Pediatr Surg. 2013;48:573-578.

20. Rollins MD, Ward RM, Jackson WD, et al. Effect of decreased parenteral soybean lipid emulsion on hepatic function in infants at risk for parenteral nutrition-associated liver disease: a pilot study. J Pediatr Surg. 2013;48:1348-1356.

21. Belkind-Gerson J, Carreon-Rodriguez A, Contreras-Ochoa CO, EstradaMondaca S, Parra-Cabrera MS. Fatty acids and neurodevelopment. $J$ Pediatr Gastroenterol Nutr. 2008;47(suppl 1):S7-S9.

22. Ward PE. Potential diagnostic aids for abnormal fatty acid metabolism in a range of neurodevelopmental disorders. Prostaglandins Leukot Essent Fatty Acids. 2000;63:65-68.

23. Dunstan JA, Mitoulas LR, Dixon G, et al. The effects of fish oil supplementation in pregnancy on breast milk fatty acid composition over the course of lactation: a randomized controlled trial. Pediatr Res. 2007;62:689-694.

24. Helland IB, Smith L, Saarem K, Saugstad OD, Drevon CA. Maternal supplementation with very-long-chain n-3 fatty acids during pregnancy and lactation augments children's IQ at 4 years of age. Pediatrics. 2003;111:e39-e44.

25. Birch EE, Garfield S, Castaneda Y, Hughbanks-Wheaton D, Uauy R, Hoffman D. Visual acuity and cognitive outcomes at 4 years of age in a double-blind, randomized trial of long-chain polyunsaturated fatty acidsupplemented infant formula. Early Hum Dev. 2007;83:279-284.

26. Fewtrell MS, Abbott RA, Kennedy K, et al. Randomized, double-blind trial of long-chain polyunsaturated fatty acid supplementation with fish oil and borage oil in preterm infants. J Pediatr. 2004;144:471-479.

27. Fleith M, Clandinin MT. Dietary PUFA for preterm and term infants: review of clinical studies. Crit Rev Food Sci Nutr. 2005;45:205-229.

28. Rydz D, Shevell MI, Majnemer A, Oskoui M. Developmental screening. $J$ Child Neurol. 2005;20:4-21.

29. Schonwald A, Huntington N, Chan E, Risko W, Bridgemohan C. Routine developmental screening implemented in urban primary care settings: more evidence of feasibility and effectiveness. Pediatrics. 2009;123:660-668.

30. Squires J, Bricker D, Potter L. Revision of a parent-completed developmental screening tool: Ages and Stages Questionnaires. J Pediatr Psychol. 1997;22:313-328.

31. Council on Children With Disabilities, Section on Developmental Behavioral Pediatrics, Bright Futures Steering Committee, Medical Home Initiatives for Children With Special Needs Project Advisory Committee. 
Identifying infants and young children with developmental disorders in the medical home: an algorithm for developmental surveillance and screening. Pediatrics. 2006;118:405-420.

32. Limbos MM, Joyce DP. Comparison of the ASQ and PEDS in screening for developmental delay in children presenting for primary care. $J$ Dev Behav Pediatr. 2011;32:499-511.

33. Sices L, Stancin T, Kirchner L, Bauchner H. PEDS and ASQ developmental screening tests may not identify the same children. Pediatrics. 2009;124:e640-e647.

34. Schonhaut L, Armijo I, Schonstedt M, Alvarez J, Cordero M. Validity of the Ages and Stages Questionnaires in term and preterm infants. Pediatrics. 2013;131:E1468-E1474.

35. Reynolds C, Kamphaus R. Behavior Assessment System for Children. 2nd ed. Circle Pines, MN: AGS Publishing; 2004.

36. Papazoglou A, Jacobson LA, Zabel T. Sensitivity of the BASC-2 Adaptive Skills Composite in detecting adaptive impairment in a clinically referred sample of children and adolescents. Clin Neuropsychologist. 2013;27:386-395.

37. Hollingshead A. Four Factor Index of Social Status. New Haven, CT: Department of Sociology, Yale University; 1975.

38. Squires J, Twombley E, Bricker D. Ages and Stages Questionnaire-3: A User's Guide. Baltimore, MD: Brookes; 2009.

39. Glascoe F. Collaborating With Parents: Using Parents' Evaluations of Developmental Status to Detect and Address Developmental Problems in Children. Nashville, TN: Ellsworth and Vandermeer Press; 1988.

40. Glascoe F. Parents' Evaluations of Developmental Status: A Method for Detecting and Addressing Developmental and Behavioral Problems in Children. Nashville, TN: Ellsworth \& Vandermeer Press; 1997.

41. Meyers C, Bour J, Sidebottom K, Murphy S. Same constructs, different results: examining the consistency of two behavior-rating scales with referred preschoolers. Psychol Schools. 2010;47:205-216.

42. Braun JM, Kalkbrenner AE, Calafat AM, et al. Impact of early-life Bisphenol A exposure on behavior and executive function in children. Pediatrics. 2011;128:873-882.

43. Rinaldi CM, Howe N. Mothers' and fathers' parenting styles and associations with toddlers' externalizing, internalizing, and adaptive behaviors. Early Child Res Q. 2012;27:266-273.
44. Davis-Bruno K, Tassinari MS. Essential fatty acid supplementation of DHA and ARA and effects on neurodevelopment across animal species: a review of the literature. Birth Defects Res B Dev Reprod Toxicol. 2011;92:240-250.

45. Lammers EM, Johnson PN, Ernst KD, et al. Association of fentanyl with neurodevelopmental outcomes in very-low-birth-weight infants. Ann Pharmacother. 2014;48(3):335-342.

46. Durrmeyer X, Hummler H, Sanchez-Luna M, et al. Two-year outcomes of a randomized controlled trial of inhaled nitric oxide in premature infants. Pediatrics. 2013;132:e695-e703.

47. Chawla S, Bapat R, Pappas A, Bara R, Zidan M, Natarajan G. Neurodevelopmental outcome of extremely premature infants exposed to incomplete, no or complete antenatal steroids. J Matern Fetal Neonatal Med. 2013;26:1542-1547.

48. Payne AH, Hintz SR, Hibbs AM, et al. Neurodevelopmental outcomes of extremely low-gestational-age neonates with low-grade periventricularintraventricular hemorrhage. JAMA Pediatr. 2013;167:451-459.

49. Kono Y, Mishina J, Yonemoto N, Kusuda S, Fujimura M. Neonatal correlates of adverse outcomes in very low-birthweight infants in the NICU Network. Pediatrics Int. 2011;53:930-935.

50. Barks JD, Liu YQ, Shangguan Y, Li J, Pfau J, Silverstein FS. Impact of indolent inflammation on neonatal hypoxic-ischemic brain injury in mice. Int J Dev Neurosci. 2008;26:57-65.

51. Xiong T, Gonzalez F, Mu DZ. An overview of risk factors for poor neurodevelopmental outcome associated with prematurity. World J Pediatr. 2012;8:293-300.

52. Serenius F, Kallen K, Blennow M, et al. Neurodevelopmental outcome in extremely preterm infants at 2.5 years after active perinatal care in Sweden. JAMA. 2013;309:1810-1820.

53. Ochiai M, Kinjo T, Takahata Y, et al. Survival and neurodevelopmental outcome of preterm infants born at 22-24 weeks of gestational age. Neonatology. 2013;105:79-84.

54. Flick RP, Katusic SK, Colligan RC, et al. Cognitive and behavioral outcomes after early exposure to anesthesia and surgery. Pediatrics. 2011;128:e1053-e1061.

55. Wilder RT, Flick RP, Sprung J, et al. Early exposure to anesthesia and learning disabilities in a population-based birth cohort. Anesthesiology. 2009; 110:796-804. 